\title{
NOTE ON AN APPARENTLY FORGOTTEN THEOREM ABOUT SOLID RIGID DYNAMICS
}

\author{
F. J. Gil Chica; M. Pérez Polo; M. Pérez Molina
}

\begin{abstract}
We re-derive a general procedure to substitute any rigid body by an equivalent system of exactly four masses, located at vertices of an irregular tetrahedron.
\end{abstract}

Keywords: Rigid solid dynamics, inertia matrix, principal axes

\section{INTRODUCTION}

In his book "An elementary treatise on the $d y$ namics of a system of rigid bodies", article N. 44, E. J. Routh [1], posed a question whose solution he only sketched: Is there a system of four masses dynamically equivalent to that of a given rigid solid? This important property seems to have been forgotten, as we have not found any proper demonstration at all. It is assumed that two geometrically different bodies are dynamically equivalent if their inertial matrices are equal. In the wellknown and respected "Lagrangian Dynamics", D. A. Wells [2], in chapter 7, stablished that different continuous mass distributions may be substituted by a variable number of puntual masses, from four to six of them, conveniently distributed, and omit the fact that any mass distribution can be substituted by exactly four masses at the vertices of an irregular tetrahedron. Moreover, D. A. Wells uses not only different number of masses, but different values for them, whilst the four equivalent masses have all the same value. The result is found nor in "A treatise on Analytical Dynamics" by L. A. Pars [3], neither in "Classical Mechanics" by $\mathrm{H}$. Goldstein [4]. Furthermore, E.T. Whittaker in his "Treatise on Analytical Dynamics of particles and rigid bodies" [5], pg. 121, states that a solid, regular tetrahedron is equimomental with four masses located at the vertices of a regular tetrahedron, plus a fifth located at the center of masses of the solid tetrahedron: "Shew that a uniform solid tetrahedron of mass $M$ is equimomental to a set of five particles, four of which are each of mass $\frac{1}{20} M$ and are situated at the vertices of the tetrahedron, while the fifth particle is at the centre of gravity of the tetrahedron and is of mass $\frac{4}{5} M$ ". The fact than only four masses are sufficient seems to have passed unnoticed. We will re-derive this elegant result in a more updated language, inserting it in the standard material about inertia matrix, principal axes and rotation.

\section{INERTIAL MATRIX OF A REGULAR TETRAHEDRON}

Let $M$ be the mass of a rigid solid. As the first step, we construct a regular tetrahedron, placing equal masses of value $m=M / 4$ at each of its vertices. Choosing the arbitrary point $(1,0,0)$ on the $x y$ plane, and rotating it $\pm 2 \pi / 3$ we complete a equilateral triangle. The fourth point is on the $z$ axis, at height $\sqrt{2}$. Now, we translate the reference system to the center of masses of the tetrahedron, whose coordinates are $(0,0, \sqrt{2} / 4)$, the side of the tetrahedron being $\sqrt{3}$. In the reference system with origin at the center of masses, the coordinates of the four vertices are:

$\left[\begin{array}{c}1 \\ 0 \\ -\sqrt{2} / 4\end{array}\right] ;\left[\begin{array}{c}-1 / 2 \\ \sqrt{3} / 2 \\ -\sqrt{2} / 4\end{array}\right] ;\left[\begin{array}{c}-1 / 2 \\ -\sqrt{3} / 2 \\ -\sqrt{2} / 4\end{array}\right] ;\left[\begin{array}{c}0 \\ 0 \\ 3 \sqrt{2} / 4\end{array}\right](1)$

from which we readily derive the inertial matrix:

$$
\mathbf{I}=\left[\begin{array}{lll}
I_{x x} & I_{x y} & I_{x z} \\
I_{y x} & I_{y y} & I_{y z} \\
I_{z x} & I_{z y} & I_{z z}
\end{array}\right]
$$

where 


$$
\begin{aligned}
& I_{x x}=\sum_{i} m_{i}\left(y_{i}^{2}+z_{i}^{2}\right) \\
& I_{y y}=\sum_{i} m_{i}\left(x_{i}^{2}+z_{i}^{2}\right) \\
& I_{z z}=\sum_{i} m_{i}\left(x_{i}^{2}+y_{i}^{2}\right) \\
& I_{x y}=I_{y x}=-\sum_{i} m_{i} x_{i} y_{i} \\
& I_{x z}=I_{z x}=-\sum_{i} m_{i} x_{i} z_{i} \\
& I_{y z}=I_{z y}=-\sum_{i} m_{i} y_{i} z_{i}
\end{aligned}
$$

Substituting Eq. (1) into Eq. (3) we have a diagonal matrix, whose principal moments are equal, having the value of $3 \mathrm{~m}$. So, the tetrahedron inertia matrix is:

$$
\mathbf{I}=\left[\begin{array}{ccc}
3 m & 0 & 0 \\
0 & 3 m & 0 \\
0 & 0 & 3 m
\end{array}\right]
$$

\section{SCALING}

Consider a generic inertia matrix for a rigid solid defined as:

$$
\mathbf{I}=\left[\begin{array}{lll}
I_{11} & I_{12} & I_{13} \\
I_{21} & I_{22} & I_{23} \\
I_{31} & I_{32} & I_{33}
\end{array}\right]
$$

calculated in a given reference system 'o'. We can find the directions of the principal axes of inertia, for which the inertia matrix is diagonal:

$$
\mathbf{I}=\left[\begin{array}{ccc}
I_{1} & 0 & 0 \\
0 & I_{2} & 0 \\
0 & 0 & I_{3}
\end{array}\right]
$$

where $I_{1}, I_{2}$ and $I_{3}$ are the principal moments of inertia.

Let us individually scale the axes of the reference system in which the tetrahedron was previosly defined. This is equivalent to simply putting the four masses in their appropiate positions. We express this scaling by:

$$
\begin{aligned}
& x^{\prime}=\alpha x \\
& y^{\prime}=\beta y \\
& z^{\prime}=\gamma z
\end{aligned}
$$

The elements out of the diagonal remain zero, while the principal moments of the tetrahedron are transformed into

$$
I_{x}^{t}=m \beta^{2} \sum_{i} y_{i}^{2}+m \gamma^{2} \sum_{i} z_{i}^{2}
$$

$$
\begin{aligned}
I_{y}^{t} & =m \alpha^{2} \sum_{i} x_{i}^{2}+m \gamma^{2} \sum_{i} z_{i}^{2} \\
I_{z}^{t} & =m \alpha^{2} \sum_{i} x_{i}^{2}+m \beta^{2} \sum_{i} y_{i}^{2}
\end{aligned}
$$

where $x_{i}, y_{i}$ and $z_{i}$ are the coordinates of the regular tetrahedron, before scaling. We are looking for $(\alpha, \beta, \gamma)$ such that

$$
\left[\begin{array}{c}
I_{x}^{t} \\
I_{y}^{t} \\
I_{z}^{t}
\end{array}\right]=\left[\begin{array}{c}
I_{1} \\
I_{2} \\
I_{3}
\end{array}\right]
$$

were $\left(I_{x}^{t}, I_{y}^{t}, I_{z}^{t}\right)$ are the principal moments of inertia of the scaled tetrahedron and $\left(I_{1}, I_{2}, I_{3}\right)$ are the principal moments of inertia of the rigid solid. From (1)-(8)-(9):

$$
\frac{3 m}{2}\left[\begin{array}{lll}
0 & 1 & 1 \\
1 & 0 & 1 \\
1 & 1 & 0
\end{array}\right]\left[\begin{array}{l}
\alpha^{2} \\
\beta^{2} \\
\gamma^{2}
\end{array}\right]=\left[\begin{array}{l}
I_{1} \\
I_{2} \\
I_{3}
\end{array}\right]
$$

whose solutions are (negative solution are discarded, as they have no physical meaning)

$$
\begin{aligned}
& \alpha=\frac{1}{\sqrt{3 m}} \sqrt{-I_{1}+I_{2}+I_{3}} \\
& \beta=\frac{1}{\sqrt{3 m}} \sqrt{I_{1}-I_{2}+I_{3}} \\
& \gamma=\frac{1}{\sqrt{3 m}} \sqrt{I_{1}+I_{2}-I_{3}}
\end{aligned}
$$

\section{ROTATION}

Our last step is rotation. Given the inertia matrix of the rigid body in a reference system 'o', in general not diagonal, it is known ([2],[4]) that there is a reference system 'p' in which the inertia matrix is diagonal. The coordinates of the material points of the solid in the original reference system, $\mathbf{r}_{o}$, are related to the coordinates in the new system 'p' by $\mathbf{r}_{o}=\mathbf{R r}_{p}$, being $\mathbf{R}$ a symmetrical matrix whose columns are the eigenvectors of the inertia matrix in reference system 'o'. But, by scaling a symmetrical tetrahedron, we constructed a non symmetrical one, whose inertia matrix equal the inertia matrix of the rigid body expressed in 'p'. So, the coordinates $\mathbf{s}_{o}$ of the vertices of the scaled tetrahedron in reference system 'o' and the coordinates of the same vertices in system 'p' are linked by the same rotation $\mathbf{R}$ :

$$
\mathbf{s}_{o}=\mathbf{R s}_{p}
$$

But we have already found $\mathbf{s}_{p}$, and so, the coordinates of the vertices of the tetrahedron in the system in which the rigid body has a (in general) non diagonal matrix are precisely $\mathbf{s}_{o}$, and are given by (12). 


\section{AN EXAMPLE}

The inertial matrix of a set of eight masses, summing $M=1$, located at the vertices of a cube of side 1 , in positions:

$$
\begin{aligned}
& {\left[\begin{array}{l}
0 \\
0 \\
0
\end{array}\right] ;\left[\begin{array}{l}
1 \\
0 \\
0
\end{array}\right] ;\left[\begin{array}{l}
1 \\
1 \\
0
\end{array}\right] ;\left[\begin{array}{l}
0 \\
1 \\
0
\end{array}\right]} \\
& {\left[\begin{array}{l}
0 \\
0 \\
1
\end{array}\right] ;\left[\begin{array}{l}
1 \\
0 \\
1
\end{array}\right] ;\left[\begin{array}{l}
1 \\
1 \\
1
\end{array}\right] ;\left[\begin{array}{l}
0 \\
1 \\
1
\end{array}\right]}
\end{aligned}
$$

is

$$
\mathbf{I}=\left[\begin{array}{ccc}
1 & -1 / 4 & -1 / 4 \\
-1 / 4 & 1 & -1 / 4 \\
-1 / 4 & -1 / 4 & 1
\end{array}\right]
$$

We will substitute this set by a dynamically equivalent set of four masses of value $m=1 / 4$. The eigenvalues and eigenvectors of the matrix are

$$
\begin{gathered}
I_{1}=0.5 ; \mathbf{u}=\left[\begin{array}{l}
0.57735 \\
0.57735 \\
0.57735
\end{array}\right] \\
I_{2}=1.25 ; \mathbf{v}=\left[\begin{array}{c}
-0.40825 \\
-0.40825 \\
0.81650
\end{array}\right] \\
I_{3}=1.25 ; \mathbf{w}=\left[\begin{array}{c}
0.70711 \\
-0.70711 \\
0
\end{array}\right]
\end{gathered}
$$

from where

$$
\mathbf{R}=\left[\begin{array}{ccc}
0.57735 & -0.40825 & 0.70711 \\
0.57735 & -0.40825 & -0.70711 \\
0.57735 & 0.81650 & 0
\end{array}\right]
$$

Note that the columns of the rotation matrix are the eigenvectors. The determinant of the matrix is +1 , given the fact that $\mathbf{R}$ is a rotation matrix. From (11), we have $\alpha=1.63300, \beta=0.81650 \mathrm{y}$ $\gamma=0.81650$. If we scale (1), the vertices of the tetrahedron become:

$$
\begin{aligned}
& {\left[\begin{array}{l}
1.63300 \\
0.00000 \\
0.28868
\end{array}\right]} \\
& {\left[\begin{array}{c}
-0.81650 \\
0.70711 \\
-0.28868
\end{array}\right]} \\
& {\left[\begin{array}{c}
-0.81650 \\
-0.70711 \\
-0.28868
\end{array}\right]} \\
& {\left[\begin{array}{l}
0.00000 \\
0.00000 \\
0.86603
\end{array}\right]}
\end{aligned}
$$

We apply the rotation $\mathbf{R}$ to these vertices, obtaining the final positions:

$$
\begin{aligned}
& {\left[\begin{array}{l}
0.73869 \\
1.14694 \\
0.94281
\end{array}\right]} \\
& {\left[\begin{array}{c}
-0.96421 \\
-0.55596 \\
0.10595
\end{array}\right]} \\
& {\left[\begin{array}{c}
-0.386855 \\
0.021397 \\
-1.048761
\end{array}\right]} \\
& {\left[\begin{array}{c}
0.61238 \\
-0.61238 \\
0.00000
\end{array}\right]}
\end{aligned}
$$

To ensure the correcteness of the procedure, we calculate the inertial matrix of the equivalent system of four equals masses of value $m=1 / 4$ :

$$
\mathbf{I}=\left[\begin{array}{ccc}
1 & -1 / 4 & -1 / 4 \\
-1 / 4 & 1 & -1 / 4 \\
-1 / 4 & -1 / 4 & 1
\end{array}\right]
$$

This matrix equals the inertial matrix of the original system of eight masses of value $m=1 / 8$

\section{CONCLUSION}

An apparently forgotten, elegant result has been re-derived, showing that any solid rigid can be replaced by an equivalent system of exactly four equal masses located at the vertices of an irregular tetrahedron. Our re-derivation has been performed in a slightly more contemporary language, linking it with a problem of scaling and rotating. We expect to enlighten undergraduate students, providing them with an additional conceptual and computational tool for the study of rigid bodies.

\section{BIBLIOGRAPHY}

[1] E. J. Routh, Dynamics of a system of rigid bodies, MacMillan and Company Ltd., 1905.

[2] D. A. Wells, Lagrangian dynamics, McGrawHill Book Company, 1967.

[3] L. A. Pars, A treatise on Analytical Dynamics, Wiley, 1965.

[4] H. G. Goldstein, Classical Mechanics, AddisonWesley, 1959.

[5] E. T. Whittaker, Treatise on Analytical Dynamics of particles and rigid bodies, Cambridge University Press, 1917. 